\title{
Fluctuations and the QCD phase diagram
}

\author{
Bernd-Jochen Schaefer ${ }^{1, * *}$ \\ ${ }^{1}$ Institut für Physik, Karl-Franzens-Universität Graz, 8010 Graz, Austria \\ In this contribution the role of quantum fluctuations for the QCD phase diagram \\ is discussed. This concerns in particular the importance of the matter back-reaction \\ to the gluonic sector. The impact of these fluctuations on the location of the con- \\ finement/deconfinement and the chiral transition lines as well as their interrelation \\ are investigated. Consequences of our findings for the size of a possible quarkyonic \\ phase and location of a critical endpoint in the phase diagram are drawn.
}

\section{INTRODUCTION}

Driven by running high-energy heavy-ion collision experiments at LHC, RHIC and the SPS and upcoming heavy-ion facilities such as FAIR, NICA and J-PARC there is a strong interest to gain a deeper understanding of the properties of QCD matter under extreme conditions. The general chiral and deconfining phase structure and in particular the possible existence of a critical endpoint (CEP) in the QCD phase diagram are much under debate, see e.g. [1]. Analyzing the relevant temperature and density region in the phase diagram is clearly a non-perturbative task and suitable methods have to be applied, e.g. [2].

For vanishing chemical potential, lattice QCD is a suitable non-perturbative technique to provide reliable insights in the QCD finite temperature transition but fails at larger densities due to the notorious fermion sign problem at non-vanishing chemical potential. Recently, impressive progress has been achieved in the framework of QCD-motivated Polyakovloop extended chiral models such as the Polyakov-Nambu-Jona-Lasinio (PNJL) [3] and the Polyakov-quark-meson (PQM) [4-6] models. In these models information about the confining glue sector of QCD is included in form of an effective Polyakov-loop potential in the spirit of a Landau-Ginzburg approach together with a coupling of the Polyakov-loop to the quarks. The parameters of the Polyakov-loop potential are extracted from pure Yang-Mills

*Electronic address: bernd-jochen.schaefer@uni-graz.at 
lattice simulations at vanishing densities.

Such models have often been investigated in mean-field approximations but the matter sector of these models has also been studied in detail beyond the mean-field level by taking into account the quark-meson quantum fluctuations mostly in a functional renormalization group (FRG) approach, see e.g. [7].

In this context, the quantum back-reaction of the matter sector to the gluonic sector in these Polyakov-loop extended models has been omitted. The Polyakov-loop potential has a parameter $T_{0}$ that is adjusted to the, for $N_{c}=3$, first-order deconfinement transition temperature of $T_{c}=270 \mathrm{MeV}$ in the pure gauge theory where no dynamical quarks are present.

In [5] the quantum back-reaction has been taken into account and acquires in the presence of dynamical quarks a quark flavor and quark chemical potential dependent transition temperature, $T_{0}\left(N_{f}, \mu\right)$, in the Polyakov-loop potential. This first solid phenomenological estimate of the $N_{f^{-}}$and $\mu$ dependence of $T_{0}$ is based on HTL/HDL perturbation theory and one-loop running of the QCD $\beta$-function. It is remarkable, that this first estimate has been confirmed by first principle QCD computations with the FRG at real and imaginary chemical potential [8] and also in a combination of the PNJL with the statistical model [9].

This already nicely demonstrates that valuable and model-parameter independent information on the QCD phase diagram can be obtained with these QCD-motivated effective models and that these models can be understood as specific controlled approximations to full dynamical QCD. Furthermore, this allows to systematically advance these models towards full dynamical QCD.

\section{BEYOND MEAN-FIELD}

The method of choice for including quantum fluctuations at non-vanishing temperature and density is the functional renormalization group (FRG) technique.

However, in a full QCD computation the resulting FRG flow equation for the effective action consists of several flow contributions and is provided by the diagrammatic form as shown in Fig. 1, see [8]. The first two loops depict the flow contributions of the full gluon $(A)$ and ghost $(C)$ propagators with a regulator denoted as a cross in the loops. These two flow diagrams represent the quantum fluctuations of the pure glue sector of QCD and generate the Polyakov-loop potential. The third loop $(\psi)$ stands for the quark fluctuations and the 
last contribution $(\phi)$ encodes the mesonic fluctuations which are generated by dynamical hadronization, see e.g. [10] for details.

We emphasize that the flow equation for the effective action is a highly non-linear coupled system. An example of this fact is given in Fig. 2 where the gluonic (and indirectly also the ghost) propagator is modified in the presence of dynamical quarks. In addition, there are also gluonic contributions to the quark and meson correlation functions. Since the first two loops generate the pure Yang-Mills Polyakov-loop potential these observations elucidate the flavor and density dependence of the $T_{0}$ parameter in the Polyakov-loop potential [2, 5]. On the other hand, if the first two diagrams, i.e., the gluon and ghost loops, are neglected in the full flow equation, the quantum dynamics of the quark-meson system is obtained. For recent works in the FRG framework see, e.g., [7]. This simple additive structure of the different contributions to the full QCD flow shows the inherent power of quark-meson-type models to be systematically improved towards full QCD within a FRG picture. Conversely, it also allows us to use directly full QCD information in connection with these effective models which further reduce their parameter dependency and enhance their predictive power. An example is the inclusion of the $N_{f}$ and $\mu$-dependence in $T_{0}$ in the Polyakov-loop potential together with the dynamics of the quark-meson sector of the PQM model in the presence of a non-trivial Polyakov-loop expectation value [6].

\section{PHASE STRUCTURE}

The resulting phase diagrams in a PQM truncation without (left panel) and with (right panel) the back-reaction of the matter sector to the glue sector, encoded in $T_{0}\left(N_{f}, \mu\right)$, are displayed in Fig. 3. The right panel shows the phase diagram with the full dynamics in the sense that the quark-meson dynamics in the presence of the Polyakov-loop background is included with the FRG together with the matter back-reaction. Comparing both phase diagrams, the grey band which corresponds to the width of the temperature derivative of the Polyakov-loop variable at $80 \%$ of its peak height, shrinks with increasing $\mu$ if the backreaction is included. As a consequence, the deconfinement transition gets sharper at larger $\mu$ and stays close to the chiral phase boundary. This difference is even more pronounced if the quantum fluctuations are neglected via a mean-field PQM calculation with a constant $T_{0}$ [11]. For physical quark masses the chiral (pseudo)critical transition lines curve downwards 
with increasing chemical potential but there is a large region in which chiral symmetry is restored and matter confined (in the statistical sense) [12]. Thus, the size of this region depends strongly on the Polyakov-loop dynamics: it has been shown in [11] within a meanfield PQM calculation with the matter back-reaction, i.e., with a running $T_{0}(\mu)$, the size of this region has considerably shrunk.

Fig. 3 highlights the importance of the matter back-reaction via a running $T_{0}(\mu)$ : with a constant $T_{0}$ (left panel) the Polyakov loops $\Phi$ and $\bar{\Phi}$ start to deviate for non-vanishing chemical potential and the widths of their temperature derivatives increase over the whole phase diagram. On the contrary in a full FRG solution where the quark-meson fluctuations and the matter back-reaction via a running $T_{0}(\mu)$ are taken into account both transition lines coincide over the whole phase diagram (right panel).

In summary, we found that within the fully coupled system, in particular, including the back-reaction of the matter fluctuations to the Yang-Mills sector, the peak locations of the order parameters for the chiral and deconfinement transitions are closely related. Of course, at large densities the present truncations without baryons becomes questionable in particular in the hadronic phase for smaller temperatures. Nevertheless, two important generic findings of these FRG analyses can be compiled: the location of a possible critical endpoint can be excluded for small chemical potentials and there is little room for a chirally symmetric and confining region in the phase diagram.

\section{Acknowledgments}

The work presented here was done in collaboration with Tina K. Herbst and Jan M. Pawlowski. The author is grateful to the organizers of the CPOD2010 and DM2010 meetings for the invitation and hospitality extended to him during his visits at the JINR in Dubna, Russia. 
[1] P. Braun-Munzinger and J. Wambach, Rev. Mod. Phys. 81 (2009) 1031; J. Wambach, arXiv:1101.4760 [hep-ph].

[2] J. M. Pawlowski, arXiv:1012.5075 [hep-ph]; R. Alkofer, M. Mitter and B.-J. Schaefer, arXiv:1101.1977 [hep-ph].

[3] P.N. Meisinger and M.C. Ogilvie, Phys. Lett. B379, 163 (1996); R.D. Pisarski, Phys. Rev. D62, 111501 (2000); K. Fukushima, Phys. Lett. B591 (2004) 277; E. Megias, E. Ruiz Arriola and L.L. Salcedo, Phys. Rev. D74 (2006) 065005; C. Ratti, M.A. Thaler and W. Weise, Phys. Rev. D73 (2006) 014019; S.K. Ghosh, T.K. Mukherjee, M.G. Mustafa and R. Ray, Phys. Rev. D73 (2006) 114007; C. Sasaki, B. Friman and K. Redlich, Phys. Rev. D75 (2007) 074013; K. Kashiwa, H. Kouno, M. Matsuzaki and M. Yahiro, Phys. Lett. B662 (2008) 26.

[4] P. Kovacs and Z. Szep, Phys. Rev. D75 (2007) 025015; G. Marko and Z. Szep, Phys. Rev. D82 (2010) 065021; H. Mao, J. Jin and M. Huang, J. Phys. G37 (2010) 035001; U. S. Gupta and V. K. Tiwari, Phys. Rev. D81 (2010) 054019.

[5] B.-J. Schaefer, J.M. Pawlowski and J. Wambach, Phys. Rev. D76 (2007) 074023.

[6] T. K. Herbst, J. M. Pawlowski and B.-J. Schaefer, Phys. Lett. B696, (2011) 58.

[7] D. U. Jungnickel and C. Wetterich, Phys. Rev. D53 (1996) 5142; B.-J. Schaefer and H. J. Pirner, Nucl. Phys. A660 (1999) 439; N. Tetradis, Nucl. Phys. A726 (2003) 93; B.J. Schaefer and J. Wambach, Nucl. Phys. A757 (2005) 479; Phys. Rev. D75 (2007) 085015; Phys. Part. Nucl. 39 (2008) 1025; V. Skokov, et al., Phys. Rev. D82 (2010) 034029; E. Nakano, et al., Phys. Lett. B682 (2010) 401; V. Skokov, B. Stokic, B. Friman and K. Redlich, Phys. Rev. C82 (2010) 015206; V. Skokov, B. Friman and K. Redlich, arXiv:1008.4570 [hep-ph].

[8] J. Braun, L.M. Haas, F. Marhauser and J.M. Pawlowski, Phys. Rev. Lett. 106 (2011) 022002.

[9] K. Fukushima, arXiv:1006.2596 [hep-ph].

[10] H. Gies and C. Wetterich, Phys. Rev. D65 (2002) 065001; J.M. Pawlowski, Annals Phys. 322 (2007) 2831; S. Floerchinger and C. Wetterich, Phys. Lett. B680 (2009) 371; J. Braun, Phys. Rev. D81 (2010) 016008.

[11] B.-J. Schaefer, M. Wagner, Phys. Rev. D79 (2009) 014018; B.-J. Schaefer, M. Wagner and J. Wambach, PoS CPOD2009 (2009) 017; Phys. Rev. D81 (2010) 074013.

[12] L. McLerran and R.D. Pisarski, Nucl. Phys. A796 (2007) 83. 


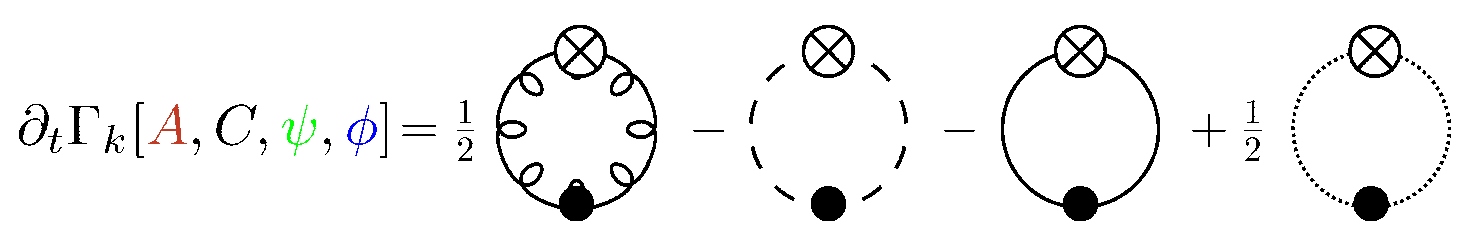

FIG. 1: Various (Gluons, ghosts, quarks and mesons) flow contributions to the QCD FRG for the effective action: The loops with the black bullet denote the corresponding full field dependent propagators and the crosses the cutoff regulator insertions. See text for details.

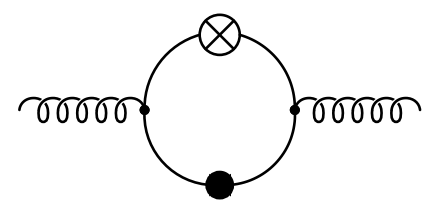

FIG. 2: Quark contributions to the flow of the gluon propagator.
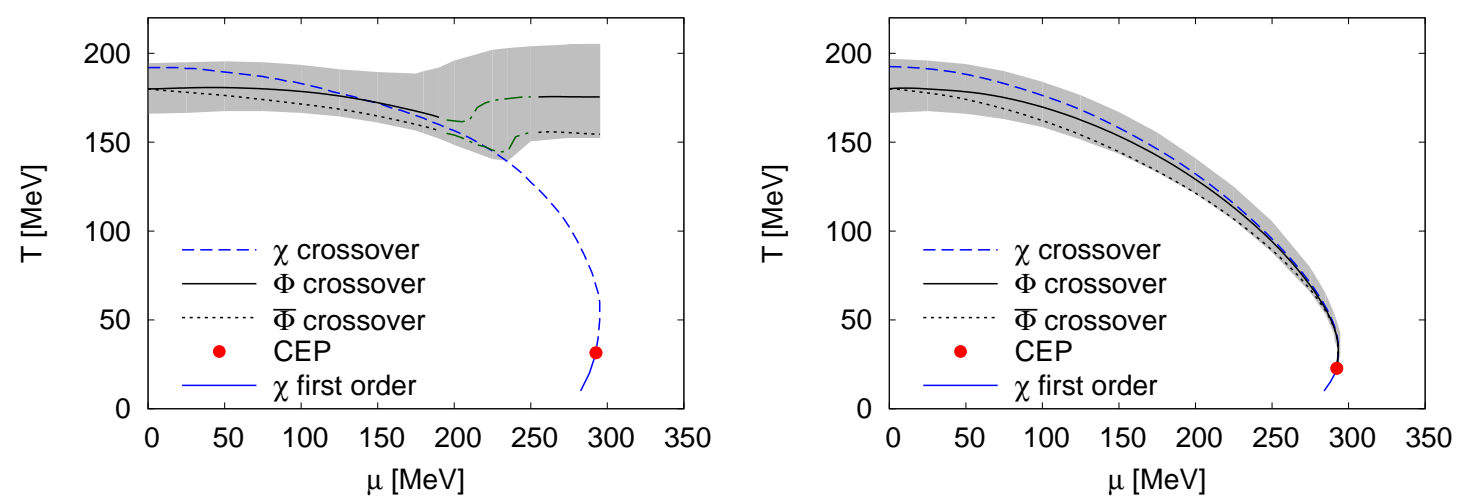

FIG. 3: The chiral $(\chi)$ and deconfinement $(\Phi, \bar{\Phi})$ phase diagrams for a constant $T_{0}=208 \mathrm{MeV}$ (left panel) and for a density dependent $T_{0}(\mu)$ (right panel). The grey band corresponds to the width of the $d \Phi / d T$ at $80 \%$ of its peak height. In the left panel, the kink behaviour in the $\Phi, \bar{\Phi}$ lines around $\mu \sim 200-250 \mathrm{MeV}$ vanishes if the used approximation of the effective potential on the mean-field solutions of the Polyakov-loop EoMs is improved. 


\section{FIGURE CAPTIONS}

1. Various (Gluons, ghosts, quarks and mesons) flow contributions to the QCD FRG for the effective action: The loops with the black bullet denote the corresponding full field dependent propagators and the crosses the cutoff regulator insertions. See text for details.

2. Quark contributions to the flow of the gluon propagator.

3. The chiral $(\chi)$ and deconfinement $(\Phi, \bar{\Phi})$ phase diagrams for a constant $T_{0}=208$ $\mathrm{MeV}$ (left panel) and for a density dependent $T_{0}(\mu)$ (right panel). The grey band corresponds to the width of the $d \Phi / d T$ at $80 \%$ of its peak height. In the left panel, the kink behaviour in the $\Phi, \bar{\Phi}$ lines around $\mu \sim 200-250 \mathrm{MeV}$ vanishes if the used approximation of the effective potential on the mean-field solutions of the Polyakovloop EoMs is improved. 